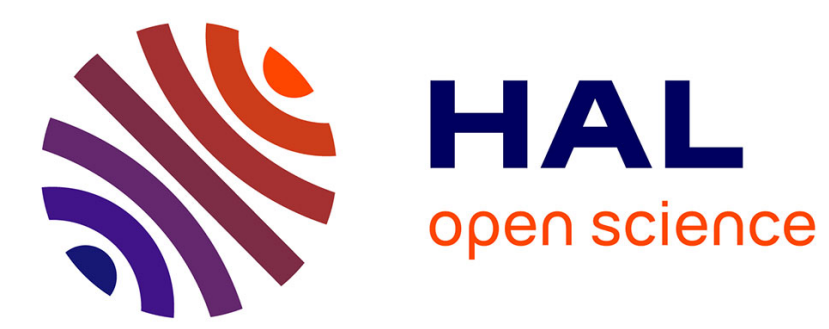

\title{
Analyse Longitudinale Des IDE En Algérie
}

Abdelmadjid Ounis, Hadjira Chigara

\section{To cite this version:}

Abdelmadjid Ounis, Hadjira Chigara. Analyse Longitudinale Des IDE En Algérie. International Journal of Economic Performance - $\quad$, , 2018 01 (02), pp.7. 10.54241/2065-000-002-

007 . hal-03455967

\section{HAL Id: hal-03455967 \\ https://hal.science/hal-03455967}

Submitted on 29 Nov 2021

HAL is a multi-disciplinary open access archive for the deposit and dissemination of scientific research documents, whether they are published or not. The documents may come from teaching and research institutions in France or abroad, or from public or private research centers.
L'archive ouverte pluridisciplinaire HAL, est destinée au dépôt et à la diffusion de documents scientifiques de niveau recherche, publiés ou non, émanant des établissements d'enseignement et de recherche français ou étrangers, des laboratoires publics ou privés. 


\section{Analyse longitudinale des IDE en Algérie}

\author{
Pr. Abdelmadjid OUNIS \\ am_ounis@yahoo.fr
}

Université de Boumerdes
Dr. Hadjira CHIGARA

chigara.hadjira@gmail.com

Université de Boumerdes

الملخص

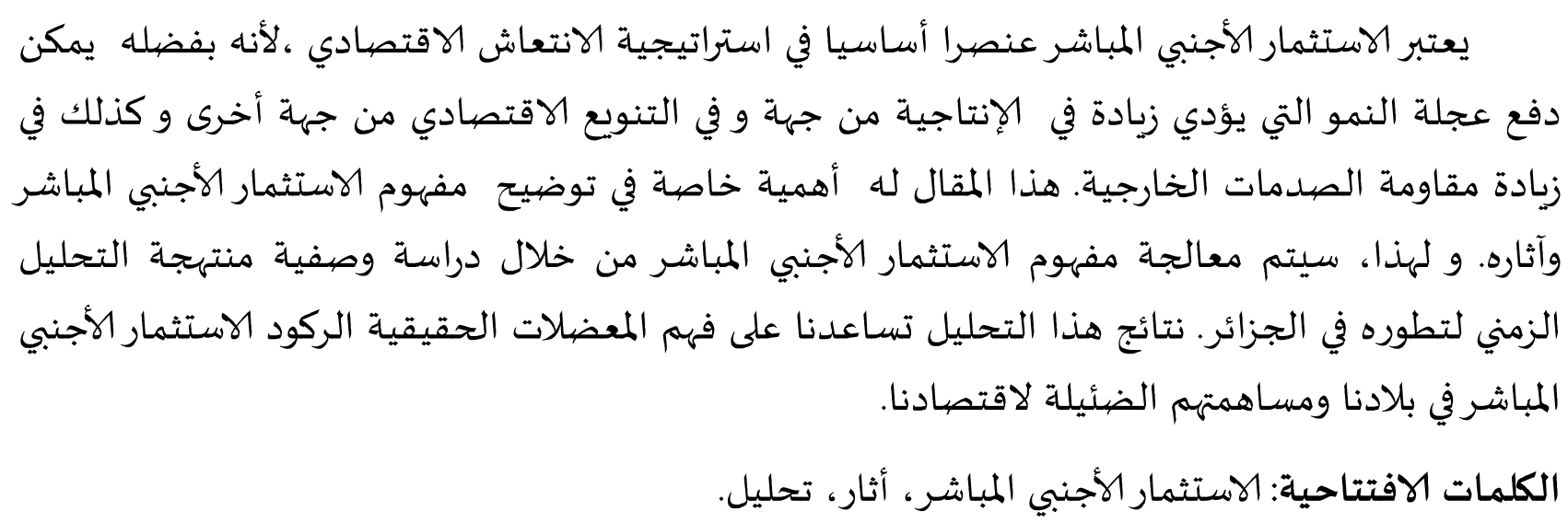

Résumé

L'investissement direct étranger est un élément essentiel dansla stratégie de la relance économique car, il peut propulser la croissance et enclencher un cercle vertueux où l'accroissement de la productivité mène à la diversification de l'économie, et à une plus grande résistance aux chocs exogènes. Ce présent article revêt une importance particulière dans l'éclairage du concept d'IDE et de ses impacts. Ainsi, il abordera grâce à une analyse descriptive longitudinale les tendances des IDE en Algérie. Les résultats de cette analyse nous permettra de comprendre les réels freins quant à la stagnation des IDE dans notre pays et à leur maigre contribution à notre économie.

Mots clés: IDE, impacts, analyse.

\section{Abstract}

Foreign direct investment is an important element in the economic recovery strategy because it can propel growth that leads to productivity rise and to economy diversification, which increase external shock resistance. This paper tries to illuminate the concept of FDI and its impacts. Thus, it addresses through a longitudinal descriptive analysis the trends of FDI in Algeria. The results of this analysis will allow us to understand the real obstacles to the stagnation of FDI in our country and their little contribution to our economy.

Key words: FDI, impacts, analysis. 


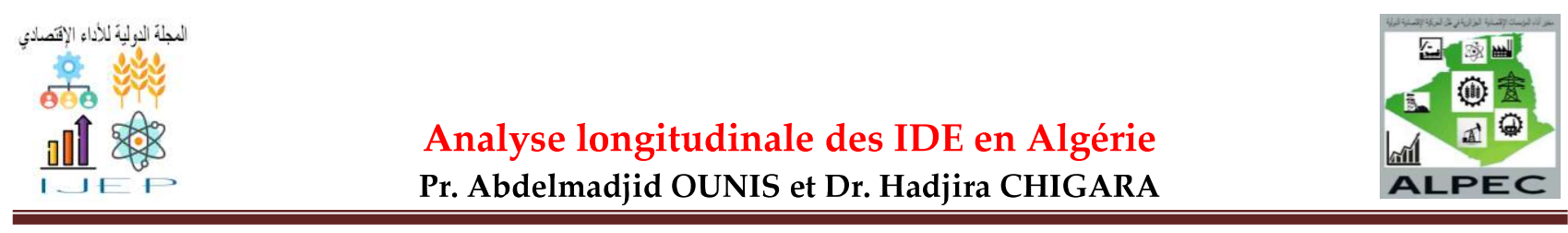

\section{INTRODUCTION}

En Algérie, la part des investissements directs étrangers reste toujours caractérisée par une faible contribution à l'économie nationale. Ce handicap persiste, même si depuis 1988, de nombreuses réformes ont été entreprises comme la stabilisation du cadre macroéconomique, la privatisation de nombreuses entreprises, la réforme du code des investissements et de la douane, la réduction du stock de la dette extérieure et des déficits budgétaires. La croissance économique quant à elle peine à s'affranchir de la pluviométrie et du prix du baril de pétrole. Cependant, en regard des contraintes structurelles qui caractérisent encore l'économie nationale, dont surtout celles liées au secteur bancaire et au climat des affaires, le commerce continue à être considéré comme étant un créneau favorable, et ce à la défaveur de l'investissement.

Dans cet article, nous tenterons de répondre à la problématique de la faiblesse des IDE en Algérie, en essayant de donner une explication quant à leur évolution qui reste toujours timide par rapport à nos voisins.

Cependant, pour pouvoir répondre à cette principale question des IDE en Algérie, nous allons tenter de les analyser à travers une approche descriptive longitudinale. De ce fait, nous allons viserdans un premier temps à clarifier le concept d'IDE, et d'un autre côté à retracer les caractéristiques des IDE en Algérie en décrivant leur tendance,

\section{Définition des IDE et leur impact sur l'économie et sur la compétitivité locale}

Nous présenterons successivement une définition des IDE, leur condition d'attractivité et leur importance.

\section{Définition des IDE}

Nombreux sont les organismes spécialisés qui ont essayé de définir l'IDE, nous en citerons quelques-unes qui nous permettront de cerner davantage cette notion.

La banque mondiale définit l'Investissement Direct Étranger comme étant «l'acquisition d'un intérêt durable dans la gestion de l'entreprise .l'investissement direct étranger suppose l'intention de détenir un actif pendant quelques années et la volonté $\mathrm{d}^{\prime}$ exercer une influence sur la gestion de cet actif $»^{1}$.

Dans sa définition des IDE, la banque mondiale met l'accent sur la détention d'un actif à l'étranger, pour lequel l'investisseur intervient dans la gestion. L'OCDE ajoute que les IDE peuvent prendre plusieurs formes, telles que créer une entité nouvelle, ou modifier le statut d'une entité existante par le biais des fusions et acquisitions.

Pour le FMI, «l'IDE est effectué dans le but d'acquérir un intérêt durable dans une entreprise exerçantes activités sur le territoire d'une économie autre que celle de l'investisseur. Le but de ce dernier étant d'avoir un pouvoir de décision effectif dans la gestion de l'entreprise»².

International Journal of Economic Performance / M'hamed Bougara-Boumerdes University / Laboratory The performance of Algerian economic institutions in light of international dynamics 


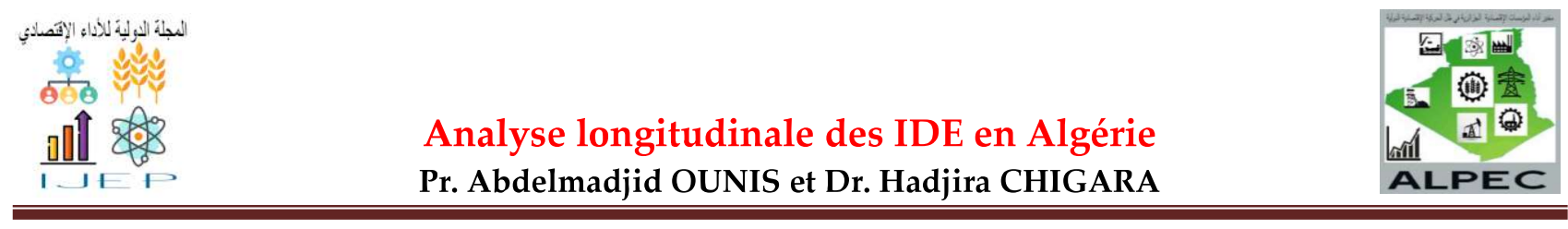

A partir de ces définitions, on peut dégager les éléments suivants:

- La notion de la durabilité d'intérêt dans l'entreprise cible,

- L'exercice de pouvoir de contrôle et d'influence sur la gestion d'une entreprise étrangère;

- Le transfert d'un savoir, savoir-faire, de compétence technologique complexes, de coordination et de gestion et de logique de production.

Par ailleurs, ce sont ces mêmes éléments qui distinguent l'IDE de tout autre investissement notamment celui des portefeuilles qui se caractérise par un investissement en actions, obligations ou autres placements financiers. Le FMI précise également que l'IDE se manifeste au niveau d'un pays étranger de l'investisseur soit par la création de nouvelles entreprises, la prise de participation dans un capital d'une entreprise existante, l'engagement dans des flux financiers entre affiliés d'un même groupe ou le réinvestissement des bénéfices à l'étranger. Ceci se manifeste généralement par la création de nouvelles filiales ou entreprises, les fusions, les licences et la franchise, sachant que ces deux dernières formes $\mathrm{d}^{\prime}$ investissement sont difficiles à quantifier car elles ne donnent pas lieux à des flux financiers.

Il y a un investissement direct étranger lorsqu'un investisseur basé dans un pays (le pays d'origine) acquiert un actif dans un pays (le pays d'accueil) avec l'intention de le gérer. L'objet de l'investissement direct est d'établir des liens économiques durables avec une entreprise. Ceci ayant pour fonction de donner la possibilité d'exercer une influence sur la gestion de ladite entreprise au moyen de:

- La création ou de l'extension d'une succursale appartenant exclusivement aux bailleurs de fonds;

- L'acquisition intégrale d'une entreprise existante;

- La participation à une entreprise nouvelle ou existante;

- Un prêt à long terme, à moins qu'il puisse être établi que cela ne permet pas à l'investisseur d'avoir un pouvoir de décision effectif dans la gestion de l'entreprise.

La notion d'intérêt durable permet de différencier, parmi les mouvements internationaux de capitaux, les IDE des investissements de portefeuille. Ces derniers sont considérés comme des placements internationaux, alors que les IDE impliquent un pouvoir de décision de l'investisseur sur l'entreprise rachetée ou construite à l'étranger. La distinction fondamentale entre IDE et investissement de portefeuille a été introduite en 1960 par S. Hymer ${ }^{3}$. Mais, l'IDE se traduit non seulement par un transfert de fonds financiers, mais aussi en général par un transfert de technologie et de capital humain (par l'intermédiaire des personnels expatriés s'impliquant dans la production à l'étranger).

On peut dire que l'investissement direct étranger est souvent lié au désir de contrôle de l'activité hors du pays d'origine, ce qui n'est pas le cas d'autres types d'investissements, notamment les investissements de placements (portefeuille). La notion de contrôle est donc déterminante. Et on peut parler d'investissement direct lorsqu'il y a contrôle de $10 \%$ ou plus des actions ordinaires ou des droits de vote. Aussi, l'investissement direct étranger peut être considéré comme une forme d'intervention et de contrôle durable d'une entreprise sur une

International Journal of Economic Performance / M'hamed Bougara-Boumerdes University / Laboratory The performance of Algerian economic institutions in light of international dynamics 


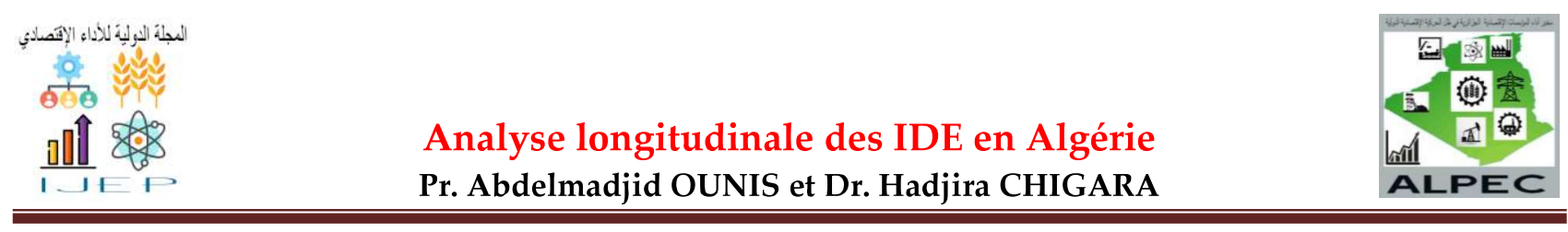

autre d'un pays différent. Les formes de l'investissement direct se distinguent de l'investissement de portefeuille parce que, principalement, ils se limitent pas à l'apport en capital financier, mais induisent surtout un transfert technologique et une capacité de gestion.

L'investissement direct étranger peut se matérialiser sous plusieurs formes:

- Un premiers investissement, qui correspond à une implantation à l'étranger et qui prend la forme de création d'une nouvelle entité (filiales, succursales) ou de l'acquisition d'une entreprise déjà existante.

- Un deuxième investissement qui se présente sous formes d'une extension, soit directement par la société mère, soit indirectement par suite des opérations menées par les entités créées lors du premier investissement.

En ce qui concerne la liaison entre le concept de firme multinationale ${ }^{4}$ et $d^{\prime}$ investissement direct étranger, que les firmes multinationales occupent une place prédominante dans l'investissement direct étranger au niveau mondial. En effet, la stratégie mondiale de ces groupes les incite à développer des investissements nécessaires au bon fonctionnement de leurs activités. Ces deux notions ne sont pas opposées et servent souvent de base aux personnes qui s'intéressent à l'internationalisation de ce type d'entreprises. Donc, on peut dire que l'IDE est à l'origine de la création des firmes multinationales ou sociétés transnationales. Une fois créé un réseau de filiales à l'étranger, l'activité de la firme multinationale ne se réduit cependant pas aux seuls IDE. Progressivement, la firme internationale va organiser au niveau international ses activités de conception, de production et de commercialisation de ses produits. Les relations entre les filiales étrangères et la maison-mère donnent alors lieu à un commerce international intra-firme de biens et de services, qui ne relève pas des IDE.

\section{Impact des IDE sur l'économie du pays d'accueil}

Plusieurs facteurs (le capital humain, l'accumulation du capital, le commerce international, la politique gouvernementale, et le transfert de la technologie), qui selon la théorie de la croissance endogène expliquent la croissance à long terme, peuvent être véhiculés par l'IDE. 5

Celui-ci, est supposé, stimuler la croissance, par la création d'avantages comparatifs dynamiques conduisant au transfert de technologie, l'accumulation du capital humain et l'intensification du commerce international. ${ }^{6}$ Ces avantages dynamiques, souvent connus sous le nom des spill-overs, sont liés les uns aux autres, complémentaires, et ne doivent pas être étudiés séparément. En effet, le gain engendré par l'IDE sur un facteur de la croissance est susceptible de stimuler le développement des autres facteurs, formant ainsi, une sorte de synergie.

Cependant, l'OCDE soutient que l'IDE peut stimuler la croissance économique en augmentant la productivité des facteurs de production. Cette vision ne fait pas l'unanimité, ainsi certain pays en voie de développement, qui sont des pays d'accueil potentiels des IDE, ont connus (ou connaissent encore parfois) des réticences face aux investissements étrangers (ce fût le cas de l'Algérie à divers périodes). Le tableau suivant, issu d'études macroempiriques, illustre les principales retombées des IDE sur l'économie des pays d'accueil:

International Journal of Economic Performance / M'hamed Bougara-Boumerdes University / Laboratory The performance of Algerian economic institutions in light of international dynamics 


Analyse longitudinale des IDE en Algérie
Pr. Abdelmadjid OUNIS et Dr. Hadjira CHIGARA

Tableau 1: Quelques études macro-empiriques des retombées de l'IDE sur la croissance économique

\begin{tabular}{|c|c|c|}
\hline Auteurs & Champs d'analyse & Principales conclusions \\
\hline $\begin{array}{c}\text { Ram et Zhang } \\
(2002)\end{array}$ & $\begin{array}{l}\text { Les entrées d'IDE dans } \\
85 \text { pays d'accueil pour } \\
\text { la période } 1990-1997 .\end{array}$ & $\begin{array}{l}\text { L'impact des entrées d'IDE sur la croissance } \\
\text { économique des pays d'accueil est } \\
\text { globalement positif, particulièrement pour le } \\
\text { sous échantillon de } 60 \text { pays les moins } \\
\text { développés. pas de complémentarité évidente } \\
\text { entre l'IDE et le capital humain local. }\end{array}$ \\
\hline $\begin{array}{c}\text { Carkovic et } \\
\text { Levine (2002) }\end{array}$ & $\begin{array}{l}\text { Les entrées d'IDE dans } \\
72 \text { pays pour la période } \\
1960-1995 .\end{array}$ & $\begin{array}{l}\text { L'IDE n'exerce pas d'effet positif robuste sur la } \\
\text { croissance économique. Léger impact positif de } \\
\text { l'IDE lorsque le pays d'accueil dispose d'un } \\
\text { système financier suffisamment développé. }\end{array}$ \\
\hline $\begin{array}{l}\text { Alfaro, Chanda, } \\
\text { Ozcan et Sayek } \\
\qquad(2001)\end{array}$ & $\begin{array}{l}\text { Les entrées d'IDE dans } \\
\text { trois échantillons de } 39 \\
\text { à } 41 \text { pays pour la } \\
\text { période } 1981-1997 .\end{array}$ & $\begin{array}{l}\text { Les pays ayant un marché de capitaux } \\
\text { suffisamment développé, bénéficient le plus des } \\
\text { retombées de l'IDE sur la croissance économique. } \\
\text { Ce résultat est robuste à différents niveaux de } \\
\text { développement des marchés de capitaux. }\end{array}$ \\
\hline Xu (2000) & $\begin{array}{l}\text { Contribution des filiales } \\
\text { étrangères à la valeur } \\
\text { ajoutée dans le PIB de } \\
40 \text { pays d'accueil pour } \\
\text { la période 1966-1994. }\end{array}$ & $\begin{array}{l}\text { L'analyse des données en panel fait ressortir } \\
\text { un impact positif de l'IDE sur la croissance de } \\
\text { la productivité totale des facteurs. la diffusion } \\
\text { de la technologie est particulièrement } \\
\text { importante pour les filiales américaines. }\end{array}$ \\
\hline De Mello (1999) & $\begin{array}{l}\text { Entrées d'IDE dans } 16 \\
\text { pays de l'OCDE et } 17 \\
\text { pays non membres pour } \\
\text { la période } 1970-1990\end{array}$ & $\begin{array}{l}\text { L'IDE stimule la croissance en augmentant la } \\
\text { production des pays de l'OCDE et en permettant } \\
\text { l'accumulation du capital dans les pays lors } \\
\text { OCDE, les retombées de l'IDE s'amenuisent dans } \\
\text { les pays de la tête de peloton technologique. }\end{array}$ \\
\hline $\begin{array}{l}\text { Borenstein, De } \\
\text { Gregorio et Lee } \\
\quad(1998)\end{array}$ & $\begin{array}{l}\text { Les flux d'IDE des pays } \\
\text { de l'OCDE vers } 69 \text { pays } \\
\text { en développement pour } \\
\text { les deux décades } 1970- \\
+1979 \text { et } 1980-1989 .\end{array}$ & $\begin{array}{l}\text { L'IDE contribue à la croissance économique } \\
\text { seulement si le pays d'accueil dispose de capacités } \\
\text { suffisantes d'absorption des technologies et des } \\
\text { compétences. L'IDE contribue relativement plus à } \\
\text { la croissance que l'investissement domestique. }\end{array}$ \\
\hline $\begin{array}{l}\text { Balasubramany } \\
\text { am, Salisu et } \\
\text { Spsford } \\
\text { (1996) }\end{array}$ & $\begin{array}{l}\text { Les entrées d'IDE dans } \\
\text { un échantillon de } 46 \\
\text { pays pour la période } \\
1970 \text { à } 1985 .\end{array}$ & $\begin{array}{l}\text { Les effets bénéfiques de l'IDE sur la croissance } \\
\text { économique sont meilleurs dans un régime orienté } \\
\text { vers l'extérieur (promotion des exportations), } \\
\text { comparativement à un régime orienté vers } \\
\text { l'intérieur (substitution des importations). }\end{array}$ \\
\hline $\begin{array}{l}\text { Blomstrom, } \\
\text { Lipsey et Zejan } \\
(1992)\end{array}$ & $\begin{array}{l}\text { Les entrées d'IDE dans un } \\
\text { échantillon de } 78 \text { pays en } \\
\text { développement pour la } \\
\text { période 19960-1985. }\end{array}$ & $\begin{array}{l}\text { Les tests de causalité de Granger font ressortir } \\
\text { un impact positif robuste de l'IDE sur la } \\
\text { croissance économique. }\end{array}$ \\
\hline
\end{tabular}

Source: Khouri, N, op.cit, p101.

International Journal of Economic Performance / M'hamed Bougara-Boumerdes University / Laboratory The performance of Algerian economic institutions in light of international dynamics 


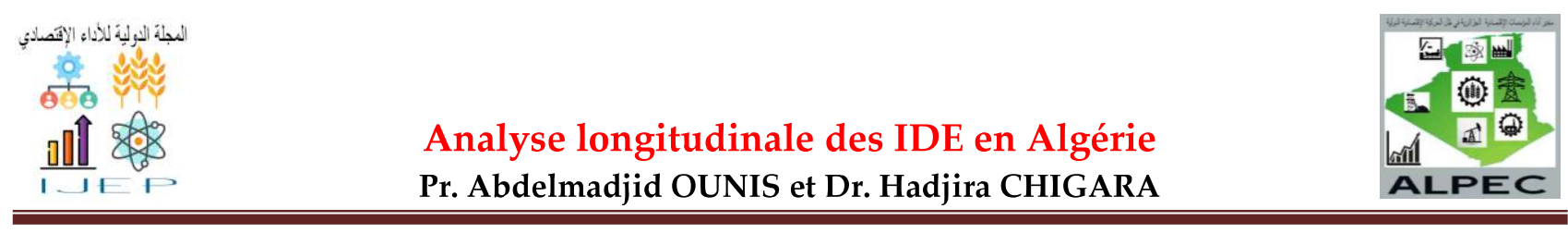

\section{Impact des IDE sur la compétitivité locale}

Un autre impact considéré d'une grande importance, réside dans la compétitivité des entreprises des pays d'accueil. Au-delà de la querelle compétitivité des nations - compétitivité des firmes, la compétitivité des nations se présente dans le contexte actuel de la mondialisation comme un facteur de compétitivité des firmes domestiques et non comme un résultat en lui-même. L'importance des investissements directs étrangers trouve sa justification dans leurs capacités à enrichir les externalités nationales offertes aux entreprises domestiques, dans leur contribution à l'amélioration de la productivité domestique et aussi dans les effets d'entraînement qu'elles exercent sur l'ensemble de l'économie. En effet, selon Dunning (1997a) ${ }^{7}$ les investissements directs étrangers pourraient avoir des répercussions favorables sur les cinq voies dont dispose la nation pour améliorer sa productivité et l'avantage comparatif de ses ressources et capacités. Ce qui permet à la nation de se procurer des ressources et des capacités à moindres coûts, d'accéléré l'apprentissage des firmes domestique, d'augmenter les standards de qualité, de stimuler l'efficience des fournisseurs et des concurrents, etc.

Ainsi, les bouleversements survenus du système techno-économique, de la mondialisation de la production, de la recherche développement et des marchés, ont eu comme corollaire l'essore des coopérations inter-entreprises et l'intensification de l'internationalisation des firmes. Ce qui suscite une question opportune sur la capacité des pays en voie de développement à tirer profit de l'avance technologique des pays industrialisés, de créer des avantages compétitifs, et d'utiliser la coopération interentreprises et l'internationalisation des firmes comme vecteurs d'apprentissages et d'acquisition de nouvelles compétences organisationnelles et technologiques. " L'aptitude de ces derniers à attirer les investissements directs étrangers, outre sa participation effective à relancer le niveau d'activité (Gannagé, 1985 ; CNUCED, 1994 ; Lall, 1995 ; OMC, 1996) et à corriger les déséquilibres macroéconomiques internes, leur permet de s'approprier des connaissances et des technologies nouvelles (Mansfield, 1961 ; Findlay, 1978 ; Hugonnier, 1984 ; Wang et Blomstrom, 1992 ; Lall, 1995 ; Muller, 1995 ; Sjoholm, 1999) et d'améliorer la productivité et la compétitivité des entreprises nationales. » (Chakroun, 2002, P.5). ${ }^{8}$

La construction de la compétitivité reste donc la principale contrainte que les pays du sud méditerranéen ont à relever. Au delà des politiques gouvernementales de mise à niveau. En effet, les pays du Sud méditerranéen ne pourront plus compté sur la seule fatalité des avantages comparatifs acquis. Par conséquent l'engagement dans des relations de partenariats avec les donneurs d'ordres européens serait une possibilité d'amélioration de la compétitivité et de mise à niveau des entreprises locales.

International Journal of Economic Performance / M'hamed Bougara-Boumerdes University / Laboratory The performance of Algerian economic institutions in light of international dynamics 


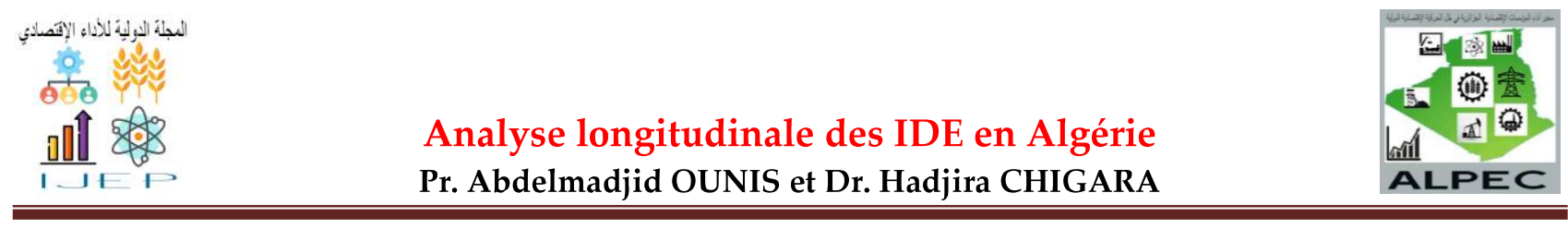

Les comportements stratégiques de coopération Nord-Sud présentent une aide au développement, et un outil efficace de « mise à niveau » des entreprises du Sud. Et « les alliances industrielles impliquent en effet la construction de mécanismes garantissant la compatibilité des processus productifs (et non seulement des produits finis), dont la mise en place de règles et plus largement d'institutions dont on peut percevoir certains éléments comme les certifications ISO 9000, les systèmes juridiques, ou les programmes globaux de «mise à niveau». Des relations de partenariat avec les firmes européennes représentent une importance particulière, d'autant plus que le contexte dans lequel va s'intégrer le tissu productif local est de plus en plus concurrentiel. Il faut croire que les facteurs organisationnels et stratégiques prendront une importance croissante.

Cependant, selon les cinq voies que Dunning à énumérer à travers lesquels les IDE améliorent la compétitivité des entreprises locales sont :

- accroître l'efficience dans le déploiement des actifs existants en adoptant des procédures de contrôle de qualité plus efficaces (...);

- innover par de nouveaux produits, processus et structures organisationnelles en améliorant le système national d'innovation (...);

- améliorer l'allocation de ses ressources et capacités, c'est-à-dire des activités moins productives vers les activités plus productives, et vers celles dans lesquels l'avantage comparatif dynamique perçu augmente ;

- accaparer de nouveaux marchés (...);

- réduire les coûts et/ou accroître la rapidité de l'ajustement structurel (...)» (Dunning, 1997, p.215).

\section{Les tendances des IDE en Algérie}

Depuis l'indépendance, l'Algérie a montré sa volonté d'attirer des flux d'IDE, ceci c'est affirmé dans les différentes lois, relatives à l'investissement. En effet, les ressources naturelles dont dispose le pays additionnées à de nombreux avantages comparatifs, tels que la proximité de l'Europe, l'infrastructure, la disponibilité de la main d'œuvre (jeune), la francophonie et les avantages fiscaux, sont des déterminants favorables. Le processus de transition d'un système centralisé à une économie de marché a imposé au gouvernement algérien, dès le début des années 1990, une politique de réformes structurelles, qui a permis le rétablissement des équilibres macro-économiques et la libéralisation de l'économie ${ }^{9}$. La privatisation des entreprises publiques et l'ouverture aux IDE ont constitué deux axes importants dans la politique économique suivie par l'Algérie depuis le début des années 2000. Certes, l'Algérie a

International Journal of Economic Performance / M'hamed Bougara-Boumerdes University / Laboratory The performance of Algerian economic institutions in light of international dynamics 
connu ces dernières années un flux d'IDE important par rapport aux années 1990, mais qui restent néanmoins insuffisants par rapport au potentiel du pays.

\section{1. Évolution des IDE en Algérie}

Bouyacoub (2007) analyse les flux d'IDE reçus en Algérie, en mettant en exergue l'existence de trois grandes périodes d'après le mouvement des flux ${ }^{10}$ :

- La période 1973-1979, qui a connu l'ouverture de secteur des hydrocarbures aux capitaux étrangers en 1971, notamment dans les branches du pétrole, du gaz naturel, du raffinage, de la production et de l'exploration. ;

- La période 1980-1995, qui a connu une absence quasi-totale des IDE. Cependant, les investissements dans le secteur des hydrocarbures ont continué à intervenir ;

- La période après 1996, qui commence avec la nouvelle législation sur la privatisation des entreprises publiques (1995) et le cadre institutionnel d'appui plus approprié d'après le nouveau code des investissements de 1993, amendé en 2001 et en 2006. Il s'agit d'une période qui a connu un développement relativement important des flux d'IDE.

Selon Cnuced, l'évolution des IDE de 1970 à 2010 est illustrée dans le graphisme suivant:

Figure1: Évolution des IDE de 1970 à 2014

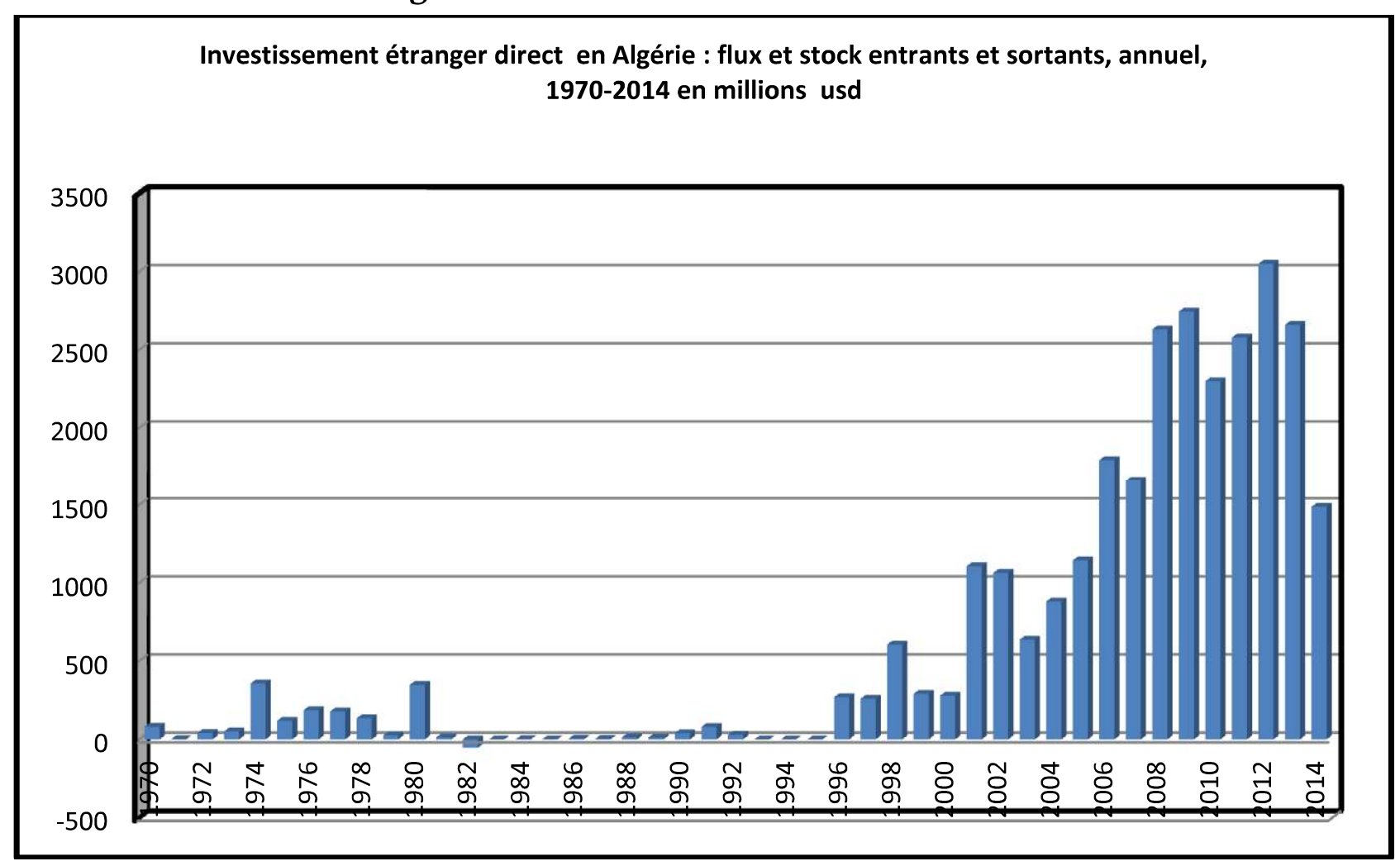

Source: Établie par nos soins, selon la base de données FDI/TNC (http://www.unctad.org//fdistatistics)

International Journal of Economic Performance / M'hamed Bougara-Boumerdes University / Laboratory The performance of Algerian economic institutions in light of international dynamics 


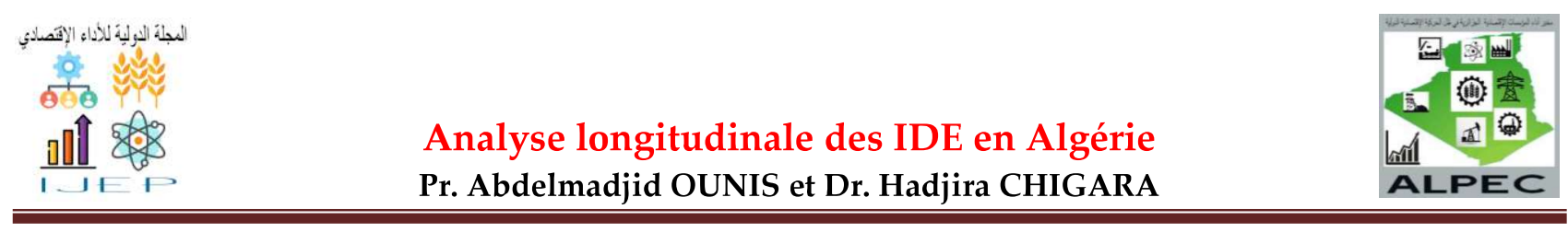

A partir du début des années 70, le pays a enregistré une profonde orientation. Ce changement s'est concrétisé par la définition d'un cadre juridique et institutionnel plus favorable à l'implantation des firmes étrangères. Dans cette perspective, l'IDE va connaître une forte croissance dans les années 70. Mais, il va enregistrer une stagnation dès le début des années 80 .

Le Graphique ci-dessus montre bien que la stagnation a duré plus d'une dizaine d'années. Cette situation coïncide avec la détérioration du cadre macroéconomique et sécuritaire. Le gouvernement a renforcé le cadre législatif avec le code d'investissement de 1993, qui n'a pas eu de succès tout de suite. Un léger regain d'intérêt des investisseurs pour le site Algérie est affiché dès la stabilisation du cadre général.

Il faut signaler qu'à partir de l'ouverture de l'industrie du pétrole et du gaz naturel en 1971, l'Algérie a attiré d'importants flux d'IDE dans ces secteurs. Notons que près de $100 \%$ des investissements sont concentrés dans le secteur des hydrocarbures, à cette période. Ceux-ci ont été massivement investis dans le raffinage, l'exploration, la production et le transport des hydrocarbures. L'attractivité de ce secteur s'est accrue suite aux chocs pétroliers des années 70 et 80 .

L'augmentation $\mathrm{du}$ prix $\mathrm{du}$ pétrole et l'interdiction faite aux grands groupes pétroliers de posséder des champs pétrolifères ont rendu d'autant plus intéressants les investissements dans les infrastructures pétrolières et gazières et permis par la même occasion de pallier au manque de ressources financières nécessaires pour l'entretien et la mise à niveau de celles-ci. Néanmoins, avant 1992, l'Algérie n'autorisait pas les sociétés étrangères à produire directement pour leur propre compte. Elles pouvaient cependant bénéficier de contrats de partage de production ou autres contrats de prestation de service avec l'entreprise publique Sonatrach. Cependant, en vertu de codes d'investissement émis en 1983 et 1986, le régime de l'Algérie l'investissement étranger était très restrictif. L'investissement étranger a été autorisé que dans des coentreprises avec des sociétés d'État, bien que le rapatriement des bénéfices ait été garanti. Le secteur de l'économie en hydrocarbures principale et beaucoup d'autres étaient hors limites. L'argent et le droit du crédit de Mars 1990 a permis la majorité à capitaux étrangers de coentreprises dans presque tous les secteurs sauf le secteur des hydrocarbures, la production d'électricité, transport ferroviaire et les télécommunications. La loi prévoyait le transfert en toute sécurité du capital et les conditions de l'arbitrage international. La loi sur les hydrocarbures de Novembre 1991 a autorisé les entreprises étrangères d'exploiter des

International Journal of Economic Performance / M'hamed Bougara-Boumerdes University / Laboratory The performance of Algerian economic institutions in light of international dynamics 
champs pétroliers existants, en partenariat avec l'entreprise pétrolière d'État. Le code des investissements d'octobre 1993 n'a pas de distinction entre les investissements réalisés par des étrangers ou des Algériens et a accordé de nouveaux investisseurs des exonérations fiscales limitées et des réductions de droits sur les marchandises importées. En 1995, le gouvernement algérien a créé l'Agence nationale de développement des investissements (Agence de Promotion, de Soutien, et de Suivi des Investissements-APSI) et les agences régionales de promotion de l'investissement pour servir un réseau régional des guichets uniques pour éliminer les couches de la bureaucratie pour les investisseurs. En 1996, APSI a approuvé 50 projets d'investissements étrangers, y compris américain (2), français (16), italien (11), espagnol (8), et allemand (4) investisseurs. En 2002, 20 entreprises à capitaux étrangers avaient été mises en place et le gouvernement s'est fixé un objectif (en 2003) pour doubler ce nombre. En 1997, l'investissement direct étranger (IDE) était de 260 millions de dollars et de 1998 à 2000 en moyenne 482 millions de dollars. En 2000, la firme allemande Henkel a acquis $60 \%$ du détergent et de l'état de produits d'entretien cabinet, Enad, et une société égyptienne a acheté une deuxième licence de téléphonie mobile GSM. En 2001, les IDE ont plus que doublé à 1196 millions de dollars principalement grâce à la privatisation et la vente d'une entreprise d'État majeur, le complexe sidérurgique d'El Hadjar, SIDER, à l'entreprise sidérurgique ISPAT les Indiens, qui a acquis la propriété à $70 \%$.

En Août 2001, le gouvernement a réorganisé les entreprises du secteur public pour faciliter l'investissement. Les 11 sociétés de portefeuille sectorielles dans lequel les entreprises économiques publiques (EPE) ont été organisées en 1996 ont été remplacées par 28 sociétés de gestion d'actionnariat et de la privatisation du Conseil national a été rebaptisé le Conseil participations de l'État. Tous les secteurs ont été ouverts à l'investissement étranger en 2001, y compris le secteur des hydrocarbures, dans lequel le gouvernement a mis contrats d'exploration pour les blocs de particuliers à l'encan. En 2003, 30 sociétés étrangères de pétrole et de gaz ont travaillé dans l'exploration en Algérie. Un plan proposé par le gouvernement d'avoir la compagnie pétrolière d'État, Sonatrach, la concurrence sur un pied d'égalité ou presque avec les compagnies pétrolières étrangères à travers la création de deux agences indépendantes, un organisme national pour gérer l'attribution des contrats en hydrocarbures (ALNAFT) et un organisme de réglementation pour les l'industrie avait-pétrole et le gaz toujours pas été votée par le parlement à la mi-2003. ${ }^{11} \mathrm{Il}$ faut noter qu'en 2003, l'Algérie est classée 74ème au niveau mondial, derrière la Tunisie 38ème, l'Égypte 58ème et le Maroc 61ème. En 2004, l'Algérie enregistre la première place de Maghreb en attirant 882 millions de dollars, contre 853

International Journal of Economic Performance / M'hamed Bougara-Boumerdes University / Laboratory The performance of Algerian economic institutions in light of international dynamics 
millions de dollars en Tunisie et 639 millions de dollars au Maroc. Malgré une évolution rapide, les stocks d'IDE restent relativement modestes en Algérie. Ils sont passés de 1561 millions de dollars en 1990 à 3647 millions en 2000 pour atteindre 7428 millions en 2004 . L'Algérie reste à la dernière position en matière de stock d'IDE dans le Maghreb. Le stock d'IDE enregistré en Algérie représente 41,3\% de celui de la Tunisie et 35,4\% de celui de l'Égypte qui a toujours occupé la première place dans ce domaine depuis $1990^{12}$. Sous l'angle des entrées de capitaux, l'année 2006 s'est particulièrement caractérisée par une forte expansion des investissements directs étrangers qui ont atteint 1,79 milliard de dollars, y compris les crédits entre entreprises apparentées, correspondant à une croissance de $66 \%$. L'analyse de la structure des investissements directs étrangers en 2006 fait apparaître un phénomène nouveau, à savoir que la part relative de ces investissements dans les secteurs hors hydrocarbures (53,02\%) dépasse celle du secteur des hydrocarbures $(46,97 \%)$.L'expansion des investissements directs étrangers est soutenue depuis 2004, leur montant de l'année 2006 constitue le triple du montant enregistré (634 millions de dollars) au cours de l'année 2003. Il est utile de rappeler que les entrées de capitaux au titre des investissements directs étrangers sont passées de 291,7 millions de dollars en 1999 à 1196,5 millions de dollars en 2001. L'important élan des investissements directs étrangers observé au cours de l'année 2006 indique l'attractivité certaine du marché algérien pour les investisseurs étrangers. En 2007, les opérateurs étrangers ont investi plus de 5,3 milliards d'euros. ${ }^{13}$ Aussi en 2008 le montant des investissements a atteint 2594 milliards de dollars.

En 2010, l'Algérie connaît un net recul des annonces d'IDE, en nombre de projets comme en montant, de même qu'une baisse sensible des flux réels d'IDE (-13\% par rapport à 2009 d'après la Banque mondiale). Les investisseurs sont vraisemblablement découragés par les nouveaux durcissements de la LFC 2009, qui donne par exemple la préférence aux entreprises algériennes dont les offres financières dépassent celles de leurs concurrents étrangers jusqu'à un surcoût de $25 \%$.

S'établissant à 1,5 milliard de dollars en 2014, les flux d'IDE en Algérie ont diminué de $-44 \%$ par rapport à 2013, dans le contexte plus large d'un recul mondial des flux d'IDE sur l'année de $-16 \%$ et au niveau régional en Afrique du Nord de $-8 \%$.

\section{Impact de la loi $49 / 51$ sur les IDE}

Dissuadés par une réglementation rigide et des procédures sur administrées, les investisseurs étrangers ne se bousculent pas au portillon. D'ailleurs, le bilan de la Conférence des Nations unies sur le commerce et le développement est sans appel. Tandis

International Journal of Economic Performance / M'hamed Bougara-Boumerdes University / Laboratory The performance of Algerian economic institutions in light of international dynamics 
que les flux mondiaux ont connu une légère reprise (5\% en 2010), le volume des IDE en Algérie en 2010 estimés à 2,291 milliards de dollars a baissé de 17\% par rapport à 2009 et a même enregistré une baisse par rapport à son niveau de 2008. Les relents de crise économique ne peuvent expliquer à eux seuls les piètres résultats enregistrés par l'Algérie, vu que le rapport de la Cnuced sur les flux d'IDE dans le monde précisent que les pays en voie de développement ont réussi dans un contexte de faible reprise à aspirer près de la moitié des flux de capitaux et qu'ils sont à l'origine d'un nombre record d'investissements, notamment en direction des pays du Sud. L'Algérie n'a donc pas été en mesure d'orienter une partie de ces capitaux au profit de son développement industriel. Cependant, Les aménagements introduits en 2009 sur le code de l'investissement et particulièrement la règle des 51/49, expliquent en partie ce recul.

Le tableau suivant illustre les montants annoncés en millions d'euros (ANIMA, 2015):

Figure 2: Montants annoncés en (M€), nombres de projets d'IDE et de partenariat en Algérie ,2008 - 2013

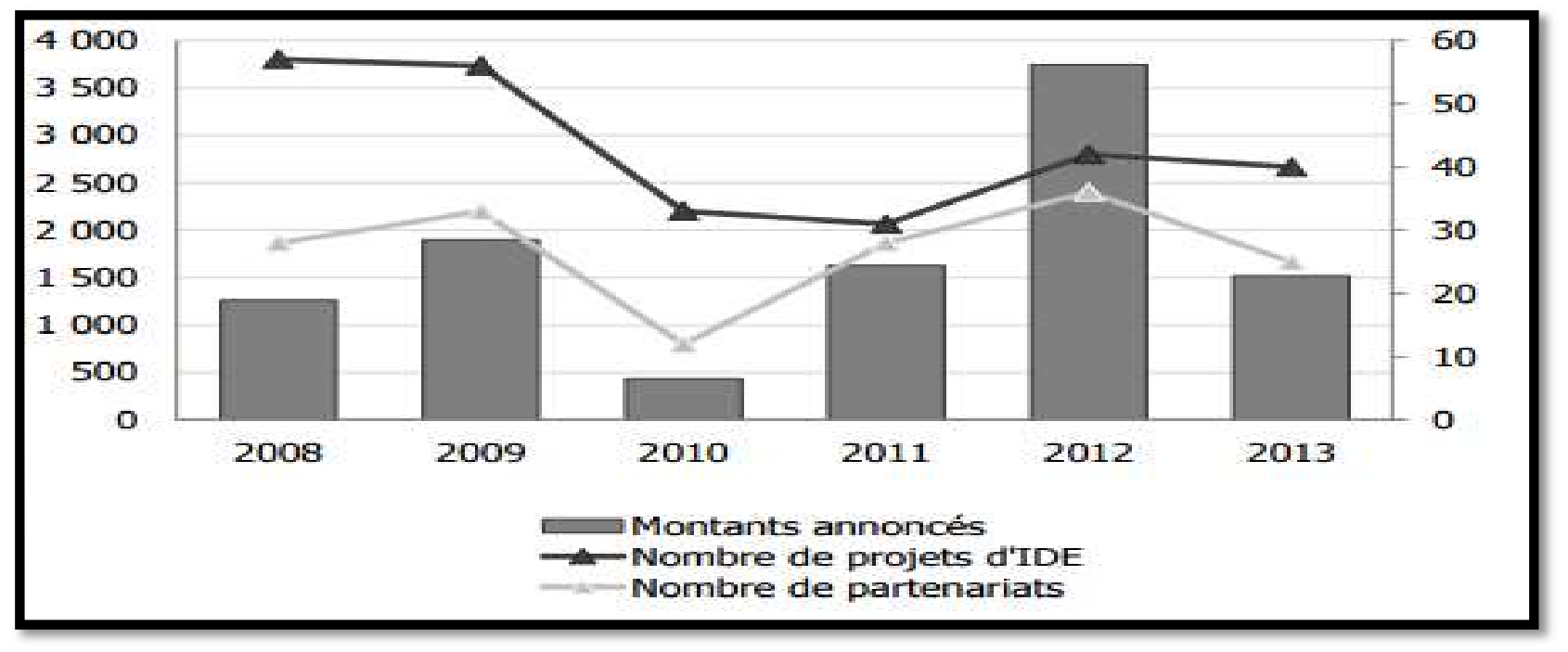

Source: ANIMA, 2015.

Nous pouvons constater qu'après l'adoption de la règle 49/51 le nombre de projets d'IDE et de partenariat a baissé énormément en 2010 contrairement à l'année précédente. Par contre en 2011 et 2012, les montants annoncés d'IDE en connu une hausse par rapport aux deux dernières années. Cela est dû à l'augmentation du nombre de projets d'investissements étrangers qui s'explique par le "niveau historiquement bas atteint en 2010. Nous pouvons noter aussi que les annonces des projets d'IDE en légère baisse en 2013 car, il faut signaler aussi qu'il n'y a pas eu de modification majeure du Code de

International Journal of Economic Performance / M'hamed Bougara-Boumerdes University / Laboratory The performance of Algerian economic institutions in light of international dynamics 
l'investissement mais à la place il ya eu une série de mesures visant à améliorer les conditions générales d'accueil des IDE. Cependant, selon le graphique sus dessus nous pouvons constater que plus de 50\% des montants annoncés en 2013 proviennent toujours du secteur énergétique. La nouvelle loi sur les hydrocarbures modifie la méthodologie utilisée pour déterminer le taux d'imposition sur les revenus pétroliers : celui-ci sera désormais calculé sur la base de la rentabilité du projet plutôt que le chiffre d'affaires, permettant ainsi de rééquilibrer les pertes potentiellement causées par des problèmes opérationnels. La loi ouvre également la voie à l'exploitation des hydrocarbures nonconventionnels et à l'utilisation de la fracturation hydraulique pour le gaz de schiste. Les projets métallurgiques ont occupé également une place importante dans les annonces d'IDE en 2013.

\section{Conclusion}

Ce papier nous a été utile pour d'un côté cerner le concept d'IDE et mettre en lumières ses principales implications pour l'économie d'accueil ainsi pour la compétitivité de ses entreprises, et d'un autre côté pour passer en revue les principales caractéristiques des IDE en Algérie ainsi que leur évolutions, et de voir l'impact de la loi 49/51 adopté par le gouvernement algérien les concernant.

On peut dire que les IDE représentent une part relativement faible de l'investissement total en Algérie. Compte tenu de leur importance pour le transfert de technologies et l'amélioration des capacités d'innovation, une politique spécifique avait été définie dans le cadre de la nouvelle stratégie industrielle. Reconnaissant le rôle déterminant des IDE pour la compétitivité et la croissance du pays, les autorités ne souhaitent cependant pas leur accorder un traitement privilégié par rapport aux investissements nationaux, jugés indispensables à la pérennité de l'économie nationale.

International Journal of Economic Performance / M'hamed Bougara-Boumerdes University / Laboratory The performance of Algerian economic institutions in light of international dynamics 


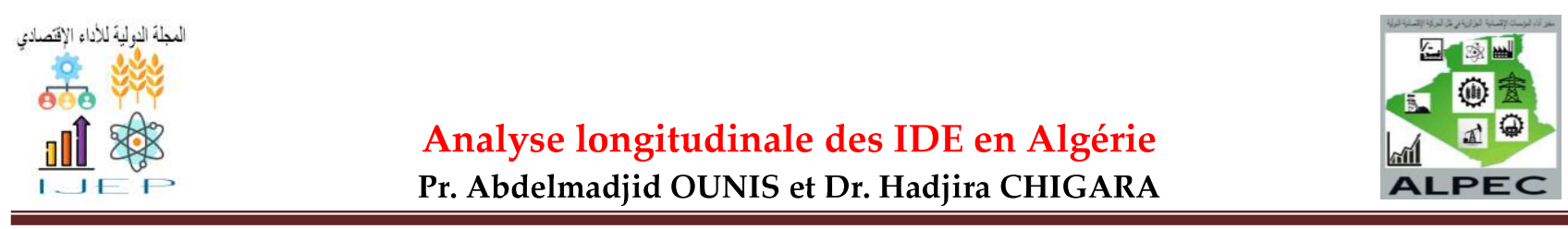

\section{Bibliographie}

1 Khouri Nabil «Les déterminants de l'investissement direct étranger dans les pays d'accueil en développement et son impact sur leur croissance économique » thèse de doctorat, ESC, Alger, 2008, p10.

2 FMI, « Manuel de la balance de paiement », 5ème édition, 2004.

3 http://fr.wikipedia.org/wiki/Investissement direct \%C3\%A0 l'\%C3\%A9tranger. Consulté le 15/05/2015.

4 Une firme multinationale peut être définie comme une organisation mondiale de production gérée par un centre de décision unique et centralisé, tirant profit de sa structuration.

5 ALAYA, M, «L'investissements directs étrangers et croissance économique : une estimation à partir d'un modèle structurel pour les pays de la rive sud de la méditerranée », 7èmes journées scientifiques du réseau " Analyse Économique et Développement de l'AUF » Université Montesquieu-Bordeaux IV, 2006, p13.

6 Bende- Nabende, A, «Foreign Direct Investment in Sub-Sahara Africa: A co-integration analysis», Economics Bulletin. Vol.6, No.4, 2002, p14.

7 Dunning J.H, « Re-evaluating the benefits of foreign direct investment » in Dunning J.H., Alliance capitalism and global business, Routledge, 1997, pp.209-233.

8 Aboutaib. Y. «Les investissements directs étrangers et la compétitivité desentreprises et de l'économie marocaine ", Séminaire doctorale, Université de Nice de sophiaAntropolis, Nice, 2004, p12.

9 Bouyacoub. A. «Le mouvement des capitaux en Algérie et principalement la question des investissements directs étrangers ». Document de travail LAREGE, , Université d'Oran, 2007,p75.

10 Ibid.

11 http://www.nationsencyclopedia.com/Africa/Algeria-FOREIGN-INVESTMENT.html. Consulté le 12/11/2015.

12 Bouyacoub,op.cit., 2007, p40.

13 ANIMA, 2015.

\section{Annexes}

Tableau 1: IDE en Algérie( flux entrants et sortants):

\begin{tabular}{|l|c|c|c|}
\hline \multicolumn{1}{|c|}{ Investissement Direct Etranger } & 2012 & 2013 & 2014 \\
\hline Flux d'IDE entrants (millions USD) & 3.052 & 2.661 & 1.488 \\
\hline Stocks d'IDE (millions USD) & 23.607 & 25.298 & 26.786 \\
\hline Nombre d'investissements greenfield*** & 18 & 16 & 13 \\
\hline IDE entrants (en \% de (a FBCF***) & 4,7 & 3,7 & 2,1 \\
\hline Stock d'IDE (en\% du PIB) & 11,4 & 12,1 & 12,5 \\
\hline
\end{tabular}

Source: CNUCED, 2015

Tableau 2: Les flux par pays et les secteurs investis en Algérie :

LES FLUXD'IDE PAR PAYSET PAR SECTEURS D'ACTIVITE

\begin{tabular}{|l|c|}
\hline \multicolumn{1}{|c|}{ Les pays investisseurs } & 2012 en $\%$ \\
\hline Koweït & 23,0 \\
\hline Espagne & 17,0 \\
\hline Egypte & 17,0 \\
\hline États-Unis & 13,0 \\
\hline France & 7,0 \\
\hline Arabie Saoudite & 6,0 \\
\hline Chine & 4,0 \\
\hline
\end{tabular}

\begin{tabular}{|l|c|}
\hline \multicolumn{1}{|c|}{ Les secteurs investis } & 2012, en \% \\
\hline Industrie & 68,8 \\
\hline BTP & 8,9 \\
\hline Transport & 8,0 \\
\hline Tourisme & 5,0 \\
\hline Services & 4,8 \\
\hline Télécommunications & 2,6 \\
\hline
\end{tabular}

Source: Agence nationale de développement de l'investissement $[Z-2016$

International Journal of Economic Performance / M'hamed Bougara-Boumerdes University / Laboratory The performance of Algerian economic institutions in light of international dynamics 